\title{
Buchbesprechungen
}

\section{Johannes Huber und Heinz Walter (Hrsg.): Der Blick auf Vater und Mutter. Wie Kinder ihre Eltern erleben (Vandenhoeck \& Rubrecht, Göttingen, 2016)} Norbert Wolff (Zürich)

Johannes Huber und Heinz Walter sammelten als Herausgeber des vorliegenden Bandes Forschungsarbeiten (hauptsächlich aus den Bereichen der pädagogischen Psychologie, Entwicklungspsychologie, Sozialwissenschaft und Psychotherapie), die vom Kind aus auf Vater und Mutter blicken. So reagierten sie auf ein Unbehagen hinsichtlich der vorherrschenden erwachsenendominierten Perspektive, die sie radikal umkehren und das kindliche Beziehungserleben zu Vater und Mutter ins Zentrum stellen. Als Leitfrage für die verschiedenen Beiträge könnte man formulieren: Wie erleben Kinder ihreVäter und Mütter und was wünschen sie sich von diesen?

Dieser Anspruch wird in den verschiedenen Beiträgen mehr oder weniger gelungen umgesetzt. Aufgrund des mit 15 Kapiteln und 18 AutorInnen sehr umfangreichen Werkes können nicht alle Facetten des Buches berücksichtigt noch kann ich allen Autoren und Autorinnen in Gänze gerecht werden, sodass sich die Rezension auf eine Auswahl beschränkt.

Huber und Walter führen in ihrem Einführungskapitel aus, warum das Erfassen der kindlichen Perspektive, die Befragung von Kindern, gar nicht so einfach ist. Im Buch werden daher von den verschiedenen Autoren und Autorinnen unterschiedliche Zugänge zur Erforschung der Beziehungsqualität, der Wahrnehmung von Vater und Mutter, der Wünsche an diese und der inneren Repräsentanzen von Vater und Mutter unternommen: teilnehmende Beobachtungen, halbstandardisierte Interviews, Erhebung von Narrativen, sogenannten «Vätergeschichten», Anwendung von projektiven Verfahren, Auswertung von Kinderzeichnungen und die Deutung von Kinderträumen. Die Vielfalt der Forschungszugänge ist dabei sehr 
eindrücklich und in der Prägnanz der Darstellung gut gelungen. Die verschiedenen Untersuchungsmethoden und theoretischen Ansätze führen in der Gesamtschau zu einem sehr differenzierten und tiefgründigen Verständnis der Bedeutung von Mutter und Vater im Laufe der kindlichen und adoleszenten Entwicklung.

Ein besonderes Augenmerk wird in diesem Sammelband auf die Bedeutung des Vaters für die kindliche Entwicklung gelegt, weil die Erforschung der VaterKind-Beziehung lange Zeit vernachlässigt («stiefmütterlich» behandelt) wurde. Inzwischen ist die Beziehung des Kindes zum Vater und dessen Einfluss für die kindliche Entwicklung zunehmend in den Fokus der Forschung gerückt. Die wichtige Bedeutung des Vaters als Vertrauensperson, Spielpartner, Rollenmodell, als Funktion in der Identitätsfindung und zur erleichterten Ablösung von der Mutter wird in verschiedenen Studien nachgewiesen.

Besonders hervorheben möchte ich in dieser Besprechung die Beiträge von einigen Psychotherapeuten zur Erschliessung der kindlichen Beziehungswahrnehmung von Vater und Mutter.

Bernd Traxl beschreibt in der psychoanalytischen Behandlung eines zu Therapiebeginn elfjährigen Jungen einfühlsam, wie stark die traumatischen Vorgeschichten von Mutter und Vater sich als Fremdkörper im Erleben des Jungen festsetzen und sich deren traumatische Erlebnisse generationsübergreifend auswirken. Die unverarbeiteten Traumata der Eltern werden verinnerlicht und über die damit einhergehenden Spannungen durch die Ausbildung von Symptomen nach aussen abgeführt. Arne Burchartz geht auf die Therapie eines zu Therapiebeginn zwölfjährigen Mädchens ein, welches starken Ängsten der werdenden Mutter ausgesetzt war und deren Ängste später, durch das anhaltende Leiden der Tochter unter Infektionskrankheiten, ihre Bestätigung fanden und zu einer starken Verstrickung von Mutter und Tochter führten, aus der der Vater ausgeschlossen wurde. Burchartz führt in seinem Bericht «Angst verstellt den Blick» nachvollziehbar aus, wie eine realistische und unabhängige Wahrnehmung der Primärobjekte beeinträchtigt wird und die inneren Repräsentanzen der Eltern voneinander abgespaltet werden müssen, da die gegensätzlichen Bilder nicht integriert werden können. Die Darstellung der Schwierigkeiten bei der Bearbeitung und Überwindung dieser lebensbehindernden Angst war sein Hauptfokus in diesem spannenden Therapiebericht. Hans Hopf berichtet in seinem Beitrag «Töchter träumen ihren Vater» anhand von drei Fallgeschichten, wie sich über das Traumgeschehen zentrale Entwicklungs- und Beziehungskonflikte mitteilen können. Neben der Funktion des Traums als verkleidete Ausdrucksform von Wünschen und Bedürfnissen, betont Hopf in seiner Einführung auch die wichtige kommunikative Funktion der Traumerzählung. In seiner Analyse des Traums eines dreijährigen Mädchens zeigt Hopf auf, wie er den verdichteten Inhalt dechiffriert, die darin enthaltene Symbolik deutet und mit dem biographischen Hintergrund des Kindes verknüpft. Eindrücklich schildert Hopf im Fall eines sehr leistungsorientierten und aggressionsgehemmten Mädchens, wie sich dieses seit seiner Geburt vergeblich um die Anerkennung seines Vaters bemühte und weshalb die Identifikation mit der Mutter für es wenig verlockend war. Die Deutung des Initialtraums und der folgenden Träume lieferten hier wichtige Hinweise auf die zugrundeliegende ödipale Dynamik.

In der Vielfalt der dargestellten Untersuchungsergebnisse zur Bedeutung der Eltern und insbesondere des Vaters für die Entwicklung von Kindern wie Heranwachsenden ist dieses Buch sehr anregend und lehrreich. Da in einigen Beiträgen Studien zu gesellschaftlichen Entwicklungen im Fokus stehen, soziologische Aspekte und pädagogische Implikationen einen breiten Raum einnehmen, machen die therapeutischen Beiträge einen nur relativ kleinen Teil des Buches aus. Persönlich hätte ich mir davon mehr gewünscht. Abgerundet wird das Buch durch Beiträge zu Kinderrechten, zur Bedeutung des eigenen Vaterbildes in einem Training für alleinerziehende Mütter sowie zur Kinderanhörung. Die Sensibilisierung für die Bedürfnisse von Kindern und die Hervorhebung der Bedeutung des Vaters für die kindliche Entwicklung ist diesem Buch gut gelungen. Es ist somit ein lesenswertes Buch für alle, die mit Kindern, Jugendlichen, Eltern und Familien arbeiten. 